Recurrent dislocation of the temporomandibular joints is uncommon, disabling when it occurs and requires urgent treatment

- The cause of the temporornandibular joint dislocation must be established prior to treatment

- Interventive surgical treatments include eminectomy and injection of Botulinum A toxin into the lateral pterygoid muscles.

\title{
Recurrent dislocation of the temporomandibular joints: a diagnostic and treatment problem
}

\author{
G. D. Wood
}

Recurrent dislocation of the temporomandibular joints is rare. Treatment of the condition is dependent on the causative diagnosis, as there are many treatment options. Three cases of recurrent temporomandibular joint dislocation treated by three different methods are used to illustrate the importance of the correct diagnostic cause before embarking on a treatment.

Dislocation of the mandible is uncommon, but is distressing and disabling and requires urgent treatment (Fig. 1). There are multifactorial causes, including trauma, hypermobile or deranged temporomandibular joints, and involuntary movements. ${ }^{1-4}$

The primary treatment of a dislocation is reduction by manual manipulation with or without sedation or general anaesthesia. Applying a downward force on the posterior teeth while pushing the mandible back over the articular eminence will return the mandible to its correct position in the glenoid fossa. All dentists should be capable of performing this procedure.

Recurrent temporomandibular joint dislocation requires preventive treatment. Current non-invasive techniques include injection of sclerosant or autologous blood around the temporomandibular joint to create fibrosis and limit jaw movement, ${ }^{3,5,6}$ a botulinum toxin A injection into the lateral pterygoid muscles ${ }^{4}$ or invasive surgery eg eminectomy or Dautrey's procedure. , $^{1,3,7,8}$

${ }^{1}$ Consultant Oral and Maxillofacial Surgeon, Department of Oral and Maxillofacial Surgery, Arrowe Park Hospital, Upton, Wirral, Merseyside, CH49 5PE

Correspondence to: Geoffrey D. Wood

E-mail:Geoff.Wood@whnt.nhs.uk

\section{Refereed Paper}

Received 04.02.02; Accepted 04.07.02

o British Dental Journal 2002; 193: 387-389
The purpose of this paper is to present three cases of recurrent temporomandibular joint dislocation treated by different techniques to demonstrate the importance of establishing the correct cause of the recurrent dislocation before embarking on corrective therapy.

\section{CASE REPORTS}

\section{Case 1}

A 76-year-old Caucasian woman with longstanding dementia and depression was referred for treatment of bilateral dislocation of the mandible. She was agitated and unable to close her mouth, speak or eat (Fig. 1). The dislocation was manually reduced without sedation and the next day using intravenous midazolam following further dislocation.

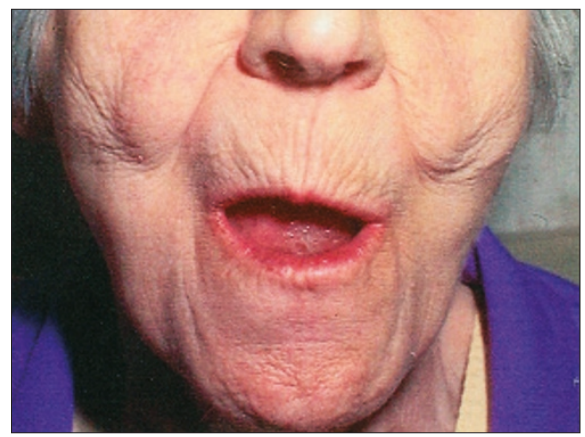

Fig. 1 A patient with bilateral dislocation of the mandible being unable to close the mouth. ascribed to tardive dystonia. lam sedation.
She had received a variety of neuroleptic drugs over 23 years and was currently taking haloperidol $10 \mathrm{mg}$ tds, plus either thioridazine $25 \mathrm{mg}$ bd. or chlorpromazine $25 \mathrm{mg}$ bd. For some time she was prone to involuntary orofacial grimacing, jaw opening and protrusion of the tongue, all

In view of the jaw dislocation her medication was stopped, and she returned to her nursing home after 4 days hospital observation. Her tardive dystonia persisted. One month later she returned with bilateral jaw dislocation, and over the following eight weeks it dislocated six times, each requiring manual reduction under midazo-

A more definitive solution was considered necessary but prior to any surgical

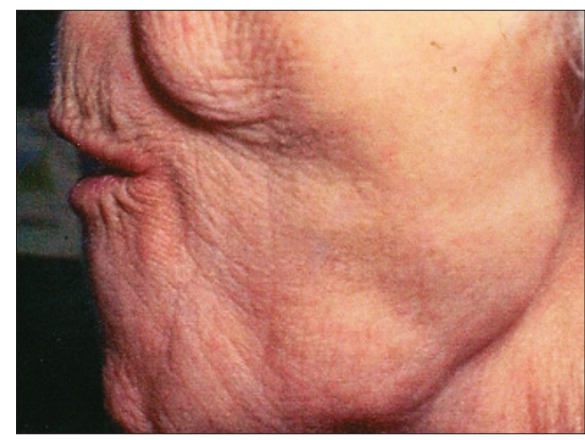

BRITISH DENTAL JOURNAL VOLUME 193 NO. 7 OCTOBER 122002 


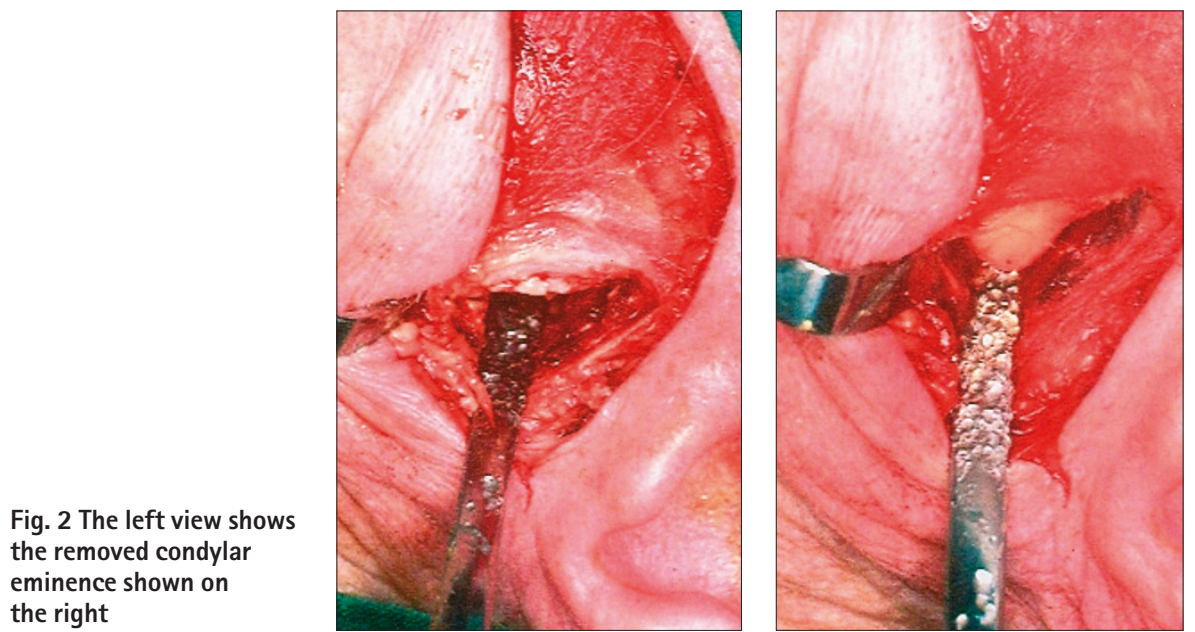

treatment botulinum toxin type A was injected into the lateral pterygoid muscles using the technique, described by Moore and Wood. ${ }^{4}$ Informed patient consent was not possible, and consent/agreement from the patient's son was obtained, as was the agreement of her general medical practitioner and a consultant in geriatric medicine.

The patient's immediate recovery was uneventful, and she went home after 4 days. No side effects were apparent and she was able to eat and speak normally for her. She remained well with no further mandibular dislocation following the procedure. The dystonic jaw opening and tongue protrusion did not recur for approximately 18 months. The procedure was repeated on two further occasions over 34 months and on each occasion the recurrent dislocation was controlled.

\section{Case 2}

A prisoner convicted of murder and sentenced to life imprisonment appeared in Court in the hope of gaining a pardon. He refused the Court's offer of parole demanding a pardon for his crime and thus was returned to prison. Later that evening his mandible dislocated and he was transferred to the prison hospital. The next day a prison visit was arranged. On examination he had a bilateral dislocation of the mandible which was confirmed by radiographic examination (lateral oblique views of mandible).

On enquiry of his previous medical history it transpired that he had been admitted to various hospitals with recurrent dislocation of the mandible from a number of prisons over his 14 years' confinement. He had been treated by manual manipulation with and without using IV sedation, intermaxillary fixation and local sclerosing solutions around the temporomandibular joints.

He had a complete dentition except for missing third molars, a Class I incisal relationship and radiographic examination confirmed well formed articular eminences in normal glenoid fossae. He was taking no medication.

It was suspected that the patient had self dislocated the mandible and he was challenged as to this diagnosis. He admitted that he had dislocated the mandible by tying string around his mandibular incisor teeth and a sudden pull accomplished a dislocated mandible. He had used the technique previously to enable him to be transferred to the prison hospital and later to a nearby local hospital to the prison where he was currently housed.

It transpired on this occasion he had some difficulty with the inmates on his block and feared for his safety. The dislocation was reduced using IV sedation and the patient transferred to another prison. An accurate record of events was entered in his prison medical notes.

\section{Case 3}

A 50-year-old Caucasian male presented with a history of recurrent dislocation of the mandible, which generally followed eating fibrous foods. He suffered from motor neurone disease, was confined to a wheel chair and was in residential care.

On the four occasions over a 3-week period during which the temporomandibular joints bilaterally dislocated, they were reduced without the need for sedation. He had dislocated on eight previous occasions over a 7-month period. The patient requested a permanent solution to the problem. A bilateral eminectomy was suggested to enable him to reduce any future dislocation himself without the need to visit hospital. The risks and benefits of the surgery were explained to him and he elected to proceed.

Bilateral eminectomies (Fig. 2) were performed under a general anaesthetic and the patient made an uneventful recovery being discharged from hospital the following day. He has not returned to hospital for reduction of a temporomandibular joint disloca- tion since the surgery and at a recent review reported he was glad to have had it. So far the surgery has been effective for 39 months.

\section{DISCUSSION}

These three cases of recurrent dislocation of the temporomandibular joints have all been successfully treated by different techniques. They illustrate the importance of correctly establishing the cause before embarking on a solution to the problem.

The patient with senile dementia (Case 1) was unable to either communicate or understand any explanation of the treatment of her dyskinesia that was causing her recurrent dislocation. Dyskinesia or dystonia is an abnormal involuntary movement with sustained and often forceful posturing or twisting of a body part. Dystonia may be persistent or intermittent, evoked only in specific situations, particularly during skilled activities using the affected part of the body, or in socially fraught situations. ${ }^{9}$ Tardive dystonia arises after treatment with neuroleptic medication, and may not abate when the drug is discontinued. Oro-facial movements are common manifestations, and jaw-opening dystonia can occur. Involuntary jaw opening which prevents proper speech and eating is highly embarrassing. Sufficiently forceful opening can result in jaw dislocation as in the case described.

Botulinum toxin type A (BtA) is a protein catalyst derived from the bacterium Clostridium botulinum. It is a powerful neurotoxin, which is selectively taken up by cholinergic (mainly neuromuscular) nerve terminals. It prevents the release of acetylcholine by damaging proteins needed to fuse vesicles with the cell membrane. If BtA is injected into a muscle it is rendered weak and atrophic within a few days or weeks. The muscle recovers over several months as the nerve terminal regenerates new protein. ${ }^{10}$ BtA is now established as the treatment of choice for a number of focal dystonias and other conditions with focal involuntary muscle activity. When used to block jaw closing and jaw opening dystonia, it is usually effective for several months after each injection. ${ }^{4,11}$

The patient made good progress with this technique avoiding open surgery and any consequent complications that she may have had difficulty in tolerating/ understanding. This technique is recommended for similar patients.

Any clinician could have been fooled into undertaking a surgical procedure on the prisoner (Case 2) should self-harm not have been established. His prison medical record confirmed that he had undergone over his 14 years of imprisonment many 
non-invasive and invasive procedures to resolve his recurrent temporomandibular joint dislocation but all appeared, not surprisingly, to have failed. He used temporomandibular joint dislocation as an escape from his various prison problems. A manual reduction of the dislocation using IV sedation and a note entry on his medical record was all that was necessary to alert another doctor should this patient present with yet another temporomandibular joint dislocation.

Self-harm by dislocating the temporomandibular joint is rare but this case illustrates the importance of establishing the cause before undertaking irreversible invasive treatment techniques.

The eminectomy has been used for many years as a reliable treatment technique for recurrent dislocation. The operation enables easy replacement of the head of the condyle into the glenoid fossa should it dislocate. The pain and discomfort of repeated bilateral mandibular condyle dislocation requiring each time a visit to the local hospital caused the patient with motor neurone disease (Case 3) to seek a more definitive solution. The eminectomy was felt to be a suitable treatment especially with the knowledge that his underlying motor neurone disease would be progressive and that it provided a permanent solution to the problem.

The described cases demonstrate the need for a comprehensive history and clinical examination to ensure planning towards the most appropriate treatment for a patient with recurrent temporomandibular joint dislocation. Invasive treatment techniques have a significant morbidity and the surgeon should be confident that the selected procedure is appropriate before embarking on it.

1. Undt G, Weichselbraun A, Wagner A, Kermer C, Roose $M$. Recurrent mandibular dislocation under neuroleptic drug therapy treated by bilateral eminectomy. J Craniomaxillofac Surg 1996; 20 217-218.

2. Raman U, Samray T. Unusual dislocation of the temporomandibular joints. A case report. Int J Oral Maxillofac Surg 1991; 20: 217-218.
3. Worsaae N, Thorn J J. Surgical versus nonsurgical treatment of unilateral dislocated low subcondylar fractures: a clinical study of 52 cases. J Oral Maxillofac Surg 1994; 52: 353-360.

4. Moore A P, Wood G D. Medical treatment of recurrent temporomandibular joint dislocation using botulinum toxin A. Br Dent J 1997; 183: 415-417.

5. Cronin A J, Llewelyn J, Hopkins R. Complications of use of carbon fibre in the tempormandibular joint: a case report. Br J Oral Maxillofac Surg 1992; 30 : 186-190.

6. Zu Y D. Clinical application of sclerosing agent in treating habitual dislocation of temporomandibula joint. Chung Hua Kou Chiang Hsueh Tsa Chih 1992; 27: 148-150, 189-190.

7. To EW. A complication of the Dautrey procedure. $\mathrm{Br}\rfloor$ Oral Maxillofac Surg 1991; 29: 100-101.

8. Srivastra D, Rajadrya M, Chaudrey M K, Savistrava J L The Dautrey procedure in recurrent dislocation: a review of 12 cases. Int J Oral Maxillofac Surg 1994; 23: 229-231.

9. Marsden CD, Quinn N P. The dystonias. Br Med J 1990; 300: 139-144.

10. Hambleton P, Moore A P. Botulinum neurotoxins: origin, structure, molecular actions and antibiodies. Brin MF, Blitzer A, Herman S, Stewart C. Oro-faciomandibular and lingual dystonia. In: Moore A P, ed. Handbook of Botulinum toxin treatment. 1st ed. Oxford: Blackwell Science Ltd, 1995: 16-27.

11. Shaari C M, Sanders I. Quantifying how location and dose of botulinum toxin injections affect muscle paralysis. Muscle Nerve 1993; 16 : 964-969. 